\title{
STABILITY OF DIFFERENTIAL INCLUSIONS: A COMPUTATIONAL APPROACH
}

\author{
VADIM AZHMYAKOV
}

Received 8 October 2004; Revised 27 December 2004; Accepted 20 April 2005

We present a technique for analysis of asymptotic stability for a class of differential inclusions. This technique is based on the Lyapunov-type theorems. The construction of the Lyapunov functions for differential inclusions is reduced to an auxiliary problem of mathematical programming, namely, to the problem of searching saddle points of a suitable function. The computational approach to the auxiliary problem contains a gradienttype algorithm for saddle-point problems. We also extend our main results to systems described by difference inclusions. The obtained numerical schemes are applied to some illustrative examples.

Copyright (C) 2006 Vadim Azhmyakov. This is an open access article distributed under the Creative Commons Attribution License, which permits unrestricted use, distribution, and reproduction in any medium, provided the original work is properly cited.

\section{Motivation and overview}

The method of Lyapunov functions is one of the most efficient methods for analyzing the stability of nonlinear dynamic systems (see, e.g., $[12,22,41]$ ). As in the case of ordinary differential equations, the main difficulty encountered in using this method for differential inclusions lies in constructing the Lyapunov function with requisite properties. The aim of our paper is to obtain an algorithm for choosing the Lyapunov function for a class of differential inclusions. Recall that differential inclusions are usual mathematical representations of control systems with ordinary differential equations (see, e.g., $[3,10,28]$ ). Let us consider the following initial-value problem $[3,7,13,21]$ :

$$
\begin{gathered}
\dot{x} \in F_{q}(x), \quad x\left(t_{0}\right)=0, \\
F_{q}(x)=\left\{y \in \mathbb{R}^{n}: y=A x, A \in \mathscr{B}_{q}\right\},
\end{gathered}
$$

where $x \in \mathbb{R}^{n}$ and $\mathscr{S}_{q}, q \in \mathbb{N}$, is the convex hull of the given real $(n \times n)$-matrices $A_{1}, \ldots$, $A_{q}$, that is,

$$
\mathscr{B}_{q}=\operatorname{co}\left(A_{1}, \ldots, A_{q}\right) \equiv\left\{A \in \mathbb{R}^{n \times n}: A=\sum_{\nu=1}^{q} \lambda_{\nu} A_{\nu}, \lambda_{\nu} \geq 0, \sum_{\nu=1}^{q} \lambda_{\nu}=1\right\} .
$$

Hindawi Publishing Corporation

Mathematical Problems in Engineering

Volume 2006, Article ID 17837, Pages 1-15

DOI 10.1155/MPE/2006/17837 
For example, the set of the initial-value problems for linear nonstationary differential equations

$$
\begin{gathered}
\dot{x}=A(t) x, \quad x(0)=0, \quad A(t)=\left(a_{i j}(t)\right)_{i, j=1}^{n}, \\
\alpha_{i j} \leq a_{i j}(t) \leq \beta_{i j}, \quad i, j=1, \ldots, n,
\end{gathered}
$$

where $\alpha_{i j}, \beta_{i j}$ are constants, can be reduced to problem (1.1).

In parallel with (1.1), we examine a more general type of the initial-value problem for differential inclusions

$$
\begin{gathered}
\dot{x} \in F(x), \quad x\left(t_{0}\right)=0, \\
F(x)=\left\{y \in \mathbb{R}^{n}: y=A x, A \in \mathscr{B}\right\},
\end{gathered}
$$

where $\mathscr{B}$ is a compactum (in general, nonconvex) in the $n^{2}$-dimensional space of real $(n \times n)$-matrices $A$.

Recall that an absolutely continuous vector function $x(\cdot)$ satisfying the condition $\dot{x}(t)$ $\in F_{q}(x(t))$ (or the condition $\dot{x}(t) \in F(x(t))$ ) almost everywhere on a considered interval of time $\left[t_{0}, t\right]$ is called a solution of the differential inclusion in (1.1) (a solution of the differential inclusion in (1.4)). Note that any solution $x(\cdot)$ of above inclusions can be continued on the whole semi-infinite axis $\left[t_{0}, \infty\right)$ (see [3]). The solution $x(\cdot) \equiv 0$ of the initial-value problem (1.1) or (1.4) is called the zero solution (or trivial solution, see, e.g., [7]). The problem of asymptotic stability of the zero solution for systems of the type (1.1) and (1.4), to which many practically important control systems can be reduced, consists of choosing a Lyapunov function $V: \mathbb{R}^{n} \rightarrow \mathbb{R}_{+}$. This function satisfies the following conditions (see $[7,13])$ :

(i) $V(0)=0, V(\cdot) \in \mathbb{C}^{1}\left(\mathbb{R}^{n}\right)$;

(ii) $V(x) \geq \varphi(|x|)>0$ on $B_{d}(0):=\left\{x \in \mathbb{R}^{n}: 0<|x|<d\right\}$ for some $d>0$ and some continuous function $\varphi:[0, \Delta) \rightarrow \mathbb{R}_{+}$.

Here $\mathbb{C}^{1}\left(\mathbb{R}^{n}\right)$ is the space of all continuously differentiable functions from $\mathbb{R}^{n}$ into $\mathbb{R}_{+}$. The Lyapunov functions $V(\cdot)$ for systems (1.1) and (1.4) can be chosen from the class of convex functions [27]. For the differential inclusions in (1.1) and in (1.4), the role of usual derivatives is played by the functions

$$
W_{q}(x)=\sup _{y \in F_{q}(x)}(\nabla V(x), y), \quad W(x)=\sup _{y \in F(x)}(\nabla V(x), y)
$$

where $\nabla V(x) y=\lim _{h \rightarrow 0_{+}} h^{-1}(V(x+h y)-V(x))$ is the Gateaux derivative (see [13]). The bracket $(\cdot, \cdot)$ denotes the inner product in $\mathbb{R}^{n}$. We call the function $W_{q}(\cdot)$ or $W(\cdot)$ the derivative of the function $V(\cdot)$ along solutions of system (1.1) or (1.4), respectively.

In this paper, we also study the question of asymptotic stability of the zero solution for systems of difference inclusions. It is common knowledge that a difference inclusion can be obtained as discretization of the corresponding differential inclusion, and provides a basis for the numerical treatment of the given differential inclusion. We refer to $[8,9,28$, 37] for details. Note that our paper is concerned only with a class of difference inclusions determined by the multifunction $F_{q}(\cdot)$. 
The problem of asymptotic stability of the zero solution for system (1.1) is closely related to the familiar problem of absolute stabilization of a nonstationary control system. Alternatively, the above problem can also be treated as a variant of the so-called robust stabilization problem. The concept of absolute stability of feedback systems dates back to Lourie and Postnikov [23]. Consider a simple model of a plant

$$
\dot{x}=\tilde{A} x+b u
$$

with nonlinear gain $u$ given in terms of the output $\sigma$ as follows:

$$
u=\phi(\sigma), \quad \sigma=(c, x) .
$$

Here $x$ (state variable), $b$, and $c$ are vectors in $\mathbb{R}^{n}, \tilde{A}$ is a matrix, and $\phi(\cdot)$ is a piecewise continuous real function. Since the nonlinearity $\phi(\cdot)$ is not a priori known in general, an interesting issue which arises is to determine conditions for stability of the given model, when $\phi(\cdot)$ is any function in a prescribed class. Thus we are led to the concept of absolute stability, which is basically stability of a whole class of systems. A decisive contribution to the Lur'e-Postnikov problem was given by Popov in a series of papers, culminating in [33]. The great advantage of the so-called frequency-domain conditions, as introduced by Popov for finite-dimensional systems, is that they are easy to check. The relationship between the frequency criteria of Popov and the existence of Lyapunov functions in Lur'e-Postnikov form (a quadratic form plus an integral of the nonlinearity) is clarified by Yakubovich [40] and by Kalman [18]. We refer to [30] for a historical survey of the problem. Some applications of the frequency techniques to the stability of delay systems are discussed in [15]. For the extensions to distributed systems, see $[6,39]$. The differential inclusion in (1.1) is equivalent to the following set of linear control systems (see [35] for details):

$$
\dot{x}=\mathscr{A} x+\sum_{\nu=1}^{q} u_{\nu}(t) A_{\nu} x, \quad 0 \leq u(t) \leq 1, \quad \sum_{\nu=1}^{q} u_{\nu}(t)=1,
$$

where the control functions $u_{\nu}(\cdot), v=1, \ldots, q$, are Lebesque measurable on each finite segment of the $t$-axis and $\mathscr{A}$ is an $(n \times n)$ matrix. Thus the problem of asymptotic stability of the zero solution for system (1.1) can be interpreted as the corresponding absolute stabilization problem for the presented set of linear control systems.

Robust stability of time-varying systems with uncertainties has received much attention since the familiar paper of Kharitonov [20]. A great amount of works is devoted to the theoretical and practical aspects of robust stability and robust stabilization of dynamical systems; see $[1,16,25,29]$ and the references therein. The so-called quadratic stabilization is a powerful approach to the problem of robust stabilization for uncertain linear systems (see, e.g., $[5,31]$ ). The problem is to construct a common quadratic Lyapunov function for all possible uncertainties. In this context, an uncertainty is meant in a deterministic sense: it arises as a result of approximation, imprecision, or imperfect knowledge introduced during the modeling procedure. In the present paper, the uncertain system is defined by a differential inclusion, the right-hand side of which is a known multifunction $F_{q}$ or $F$. Quadratic stability dates back to the pioneering work of Lyapunov 
[24] who established that the existence of a quadratic Lyapunov function is a necessary and sufficient condition for asymptotic stability of a linear system. Quadratic Lyapunov functions are often the first resort in the analysis of nonlinear systems and much work on absolute stability is based on quadratic Lyapunov functions. We refer to $[17,36]$ for some new directions in the area of quadratic stability and quadratic stabilization. The formulated problem of asymptotic stability of the zero solution for system (1.1) can also be considered in the framework of quadratic stabilization.

The aim of this paper is to propose a new numerical approach to the described problem of asymptotic stability for systems (1.1) and (1.4) and to the corresponding robust stabilization problem. Moreover, we also investigate the question of asymptotic stability for the linear "nonconvex" system (1.4) (Theorems 2.4 and 2.5). Using these new theoretical results, one can construct the Lyapunov function for a suitable system (1.1) in place of the corresponding problem for the more general system (1.4). Note that the problem of asymptotic stability of the zero solution for system (1.4) is not from the class of quadratic stabilization problems. In the present paper, we do not consider any standard techniques from the theory of absolute stability or robust stability (e.g., Popov-type criteria, Brockett technique, stability radius, LMI-based methods, Riccati equation approach, and the like). We apply some results from the classical stability theory for differential inclusions (see, e.g., [7, 12, 13]). Moreover, we discuss a relaxation of the usual stability concept [7]. In contrast to the quadratic stabilization, we construct polynomial Lyapunov functions for system (1.1) and for the corresponding discrete system. This approach makes it possible to reduce the problem of choosing the Lyapunov function to an auxiliary problem of mathematical programming, namely, to a saddle points problem (Theorems 3.1 and 4.1). We solve this saddle points problem by application of a gradient-type method.

The remainder of the paper is organized as follows. Section 2 contains some basic facts about the asymptotic stability. In Section 3, we propose a constructive approach to the stability problem and establish a relation between stability of systems given above and an auxiliary saddle points problem. Section 4 contains the related results for a class of difference inclusions. In Section 5, we apply a gradient-type method to the auxiliary saddle points problem and present an algorithm for constructing the Lyapunov functions. Finally, we consider two illustrative examples.

\section{Mathematical preliminaries}

First let us discuss some definitions and theoretical results in connection with the stability theory for differential inclusions (see $[3,7,13])$.

Definition 2.1. The zero solution $x(\cdot) \equiv 0$ of the initial-value problem (1.1) (or (1.4)) is called asymptotically stable if

(i) for any $\epsilon>0$, there exists $\delta(\epsilon)>0$ such that for each solution $\tilde{x}(\cdot)$ of the differential inclusion in (1.1) (or in (1.4)), the inequality $\|\tilde{x}(t)\|<\epsilon$ holds for all $t \geq t_{0}$, only if $\left\|\tilde{x}\left(t_{0}\right)\right\|<\delta(\epsilon)$;

(ii) there exists $\Delta>0$ such that for any solution $\tilde{x}(\cdot)$ of the differential inclusion in (1.1) (or in (1.4)) with $\left\|\tilde{x}\left(t_{0}\right)\right\|<\Delta$, the following limiting relation $\lim _{t \rightarrow \infty} \tilde{x}(t)=0$ holds. 
The zero solution $x(\cdot) \equiv 0$ is said to be weakly asymptotically stable if the above definition holds with "for each" replaced by "for some." For the classical Lyapunov-type stability theorems, see $[7,13]$. Let us present two special stability theorems for the given system (1.4) [27].

Theorem 2.2. For the zero solution $x(\cdot) \equiv 0$ of problem (1.4) to be asymptotically stable, it is necessary and sufficient that there exists a strictly convex, homogeneous (of second order) Lyapunov function $V(\cdot)$ of a quasiquadratic form, namely,

$$
\begin{gathered}
V(x)=x^{T} \mathscr{L}(x) x, \quad V(0)=0, \quad \mathscr{L}(x)=\left(l_{i, j}(x)\right)_{i, j=1}^{n}, \\
\mathscr{L}^{T}(x)=\mathscr{L}(x)=\mathscr{L}(\tau x), \quad x \neq 0, \tau \neq 0,
\end{gathered}
$$

whose derivative along solutions of system (1.4) satisfies the inequality

$$
W(x) \leq-\gamma\|x\|^{2}, \quad \gamma>0 .
$$

Note that Theorem 2.2 is a variant of the converse theorems of Lyapunov's direct method for differential inclusions [22]. In parallel with the quasiquadratic Lyapunov functions, one can also consider the Lyapunov functions from some other classes of functions, for one, from the class of homogeneous forms [27].

Theorem 2.3. The zero solution $x(\cdot) \equiv 0$ of problem (1.4) is asymptotically stable if and only if there exists a Lyapunov function in the class of homogeneous forms of order $2 p, p \in \mathbb{N}$,

$$
V_{m, p}(l, x)=\sum_{i=1}^{m}\left(l_{i}, x\right)^{2 p}
$$

where $l_{i} \in \mathbb{R}^{n}, i=1, \ldots, m$ are constant vectors with

$$
\operatorname{rank} \mathscr{L}=n \leq m, \quad \mathscr{L}=\left(l_{1}, \ldots, l_{m}\right)^{T},
$$

such that for its derivative

$$
W_{m, p}(l, x)=2 p \sup _{y \in F(x)}\left\{\sum_{i=1}^{m}\left(l_{i}, x\right)^{2 p-1}\left(l_{i}, y\right)\right\}
$$

along solutions of system (1.4), the inequality

$$
W_{m, p}(l, x) \leq-\gamma\|x\|^{2 p}, \quad \gamma>0
$$

is satisfied with an integer $p \geq 1$.

Since (1.1) is a special case of (1.4), Theorems 2.2 and 2.3 provide the necessary and sufficient conditions of the asymptotic stability of the zero solution $x \equiv 0$ for systems (1.1) and (1.4). Evidently, the parameters determining the class of Lyapunov functions $V_{m, p}(\cdot, \cdot)$ are the components of the vectors $l_{i}, i=1, \ldots, m$, and the numbers $m$ and $p$. As is shown in [27], the Lyapunov functions in Theorems 2.2 and 2.3 can be chosen from the class of convex (with respect to $x$ ) functions. 
The next result establishes a useful link between problem (1.4) and an approximate initial-value problem.

Theorem 2.4. The zero solution $x(\cdot) \equiv 0$ of problem (1.4) is asymptotically stable if and only if the zero solution of the problem

$$
\begin{gathered}
\dot{x} \in F_{c}(x), \quad x\left(t_{0}\right)=0, \\
F_{c}(x)=\left\{y \in \mathbb{R}^{n}: y=A x, A \in \operatorname{co}(\mathscr{B})\right\}
\end{gathered}
$$

is asymptotically stable.

Proof. Sufficiency of the assertion follows from the inclusion $F(x) \subseteq F_{c}(x)$. Necessity follows from the equivalence of the closure of the solution set of problem (1.4) and the solutions set of problem $(2.7)$ (see $[3,13])$.

Since a convex compactum co( $\mathscr{B})$ can be approximated by convex polyhedrons $\mathscr{B}_{q}$, we have the following result.

Theorem 2.5. For the zero solution $x(\cdot) \equiv 0$ of problem (1.4) to be asymptotically stable, it is necessary and sufficient that there exists a number $q \geq 1$ and an initial-value problem (1.1) whose zero solution $x(\cdot) \equiv 0$ is asymptotically stable and

$$
F_{c}(x) \subseteq F_{q}(x) .
$$

Proof. Let $q \in \mathbb{N}$ be a number such that $F_{c}(x) \subset F_{q}(x)$. We assume that the zero solution $x(\cdot) \equiv 0$ of $(1.1)$ is asymptotically stable. The asymptotic stability of the zero solution of the initial-value problem (2.7) is an immediate consequence of (2.8). By Theorem 2.4, the zero solution of problem (1.4) is asymptotically stable too.

Now we assume that the zero solution $x(\cdot) \equiv 0$ of problem (1.4) is asymptotically stable. It follows from the results of $[3,12]$ that there exists some $\epsilon>0$ such that the zero solution of the initial-value problem

$$
\begin{gathered}
\dot{x} \in F_{\epsilon}(x), \quad x\left(t_{0}\right)=0, \\
F_{\epsilon}(x)=\left\{y \in \mathbb{R}^{n}: y=A x, A \in \mathscr{B}_{\epsilon}\right\},
\end{gathered}
$$

where $\mathscr{B}_{\epsilon}$ is a compactum and $\mathscr{B} \subset \mathscr{B}_{\epsilon}$, is asymptotically stable. This implies that the zero solution of the problem

$$
\begin{gathered}
\dot{x} \in F_{c, \epsilon}(x), \quad x\left(t_{0}\right)=0, \\
F_{c, \epsilon}(x)=\left\{y \in \mathbb{R}^{n}: y=A x, A \in \operatorname{co}\left(\mathscr{B}_{\epsilon}\right)\right\}
\end{gathered}
$$

is asymptotically stable (see Theorem 2.4). Then there exists a number $q \in \mathbb{N}$ such that

$$
\operatorname{co}(\mathscr{B}) \subset \operatorname{co}\left(A_{1}, \ldots, A_{q}\right), \quad A_{s} \in \operatorname{co}\left(\mathscr{B}_{\epsilon}\right), \quad s=1, \ldots, q
$$

We have

$$
\operatorname{co}\left(A_{1}, \ldots, A_{q}\right) \subset \operatorname{co}\left(\mathscr{B}_{\epsilon}\right), \quad A_{s} \in \operatorname{co}\left(\mathscr{B}_{\epsilon}\right), \quad s=1, \ldots, q
$$


This means that there is a number $q \geq 1$ such that the solution $x(\cdot) \equiv 0$ of $(1.1)$ is asymptotically stable and condition (2.8) holds.

In a similar way, one can formulate the corresponding results for week asymptotically stable systems.

Theorem 2.6. The foregoing Theorems 2.2 and 2.3 hold with "asymptotically stable" replaced by "week asymptotically stable" and with derivatives $W(\cdot), W_{m, p}(\cdot, \cdot)$ replaced by functions

$$
\begin{gathered}
W^{-}(x):=\inf _{y \in F(x)}(\nabla V(x), y), \\
W_{m, p}^{-}(l, x):=\inf _{y \in F(x)}\left(\nabla V_{m, p}(l, x) y\right)=2 p \inf _{y \in F(x)}\left\{\left(l_{i}, x\right)^{2 p-1}\left(l_{i}, y\right)\right\},
\end{gathered}
$$

respectively.

Theorem 2.6 can be proved in the same way as the above-mentioned Theorems 2.2 and 2.3.

We will touch briefly on the problem of stabilization for the control system

$$
\dot{x} \in Q(x), \quad Q(x)=f(t, x, U), \quad 0 \in Q(0), \quad x\left(t_{0}\right)=0,
$$

where $f: \mathbb{R} \times \mathbb{R}^{n} \times \mathbb{R}^{m}, x$ is the state variable, and $U$ is a control region. The asymptotic stability in this case means that if $\left\|x\left(t_{0}\right)\right\|<\delta$, then the trajectory $x(\cdot)$ satisfies the inequality $\|x(t)\|<\epsilon\left(t_{0} \leq t<\infty\right)$ for every admissible control function $u(\cdot), u(t) \in U$. The weak asymptotic stability means the same inequality holds only for some admissible controls.

\section{Lyapunov functions and saddle points problem}

The Lyapunov function from Theorem 2.3 has the polynomial form

$$
V_{m, p}(l, x)=\sum_{i=1}^{m}\left(\sum_{j=1}^{n} l_{i}^{j} x_{j}\right)^{2 p}, \quad p \geq 1,
$$

or equivalently

$$
V_{p}(z, x)=\sum_{r=1}^{N(p)} z_{r} \psi_{r}(x)=(z, \psi(x)),
$$

where $\psi_{r}(x), r=1, \ldots, N(p)$, are standard monomials of degree $2 p \geq n$,

$$
\begin{gathered}
\psi(x)=x_{1}^{k_{1 r}} \cdots \cdots x_{n}^{k_{n r}} \\
\sum_{i=1}^{n} k_{i r}=2 p, \quad k_{i r} \in \mathbb{N}, r=1, \ldots, N(p),
\end{gathered}
$$

$z \in G_{z} \subset \mathbb{R}^{N(p)}$ are coefficients of monomials and $N(p)=C_{n+2 p-1}^{2 p}$ is the number of monomials. The derivatives $W_{p, q}(z, x)$ and $W_{p, q}^{-}(z, x)$ of the function $V_{p}(\cdot, \cdot)$ along solutions 
of the given system (1.1) have the form

$$
\begin{aligned}
& W_{p, q}(z, x)=\sup _{y \in F_{q}(x)}\left(z, \frac{\partial \psi(x)}{\partial x} y\right)=\max _{\lambda \in \Theta} \sum_{\nu=1}^{q} \lambda_{\nu}\left(z, \frac{\partial \psi(x)}{\partial x} A_{\nu} x\right), \\
& W_{p, q}^{-}(z, x)=\inf _{y \in F_{q}(x)}\left(z, \frac{\partial \psi(x)}{\partial x} y\right)=\min _{\lambda \in \Theta} \sum_{\nu=1}^{q} \lambda_{\nu}\left(z, \frac{\partial \psi(x)}{\partial x} A_{\nu} x\right),
\end{aligned}
$$

where $\lambda:=\left(\lambda_{1}, \ldots, \lambda_{q}\right)^{T}, \Theta:=\left\{\lambda \in \mathbb{R}^{q}: \sum_{j=1}^{q} \lambda_{j}=1, \lambda_{j} \geq 0\right\}$, and

$$
\frac{\partial \psi(x)}{\partial x}=\left(\frac{\partial \psi_{r}(x)}{\partial x_{i}}\right), \quad i=1, \ldots, n, r=1, \ldots, N(p)
$$

is an $(N(p) \times n)$-matrix. We will use the following notation: $\bar{x}:=0$.

Now we consider the bounded region $G_{x}:=\left\{x \in \mathbb{R}^{n} \mid\|x\| \leq \Gamma_{x}\right\}$ and investigate the asymptotic stability of system (1.1) for $x \in G_{x}$. Note that under the above-presented condition (2.8) Theorems 2.4 and 2.5 make it possible to reduce the question of asymptotic stability of system (1.4) to the same question for system (1.1). Therefore, we give the constructive characterization of condition (2.6) in Theorem 2.3 only for the initial-value problem (1.1).

Theorem 3.1. Let $V_{p}(\cdot, \cdot)$ be the Lyapunov function from the class of homogeneous forms of order $2 p, p \in \mathbb{N}$, for system (1.1). The inequality

$$
W_{p, q}(z, x) \leq-\gamma\|x\|^{2 p}, \quad \gamma>0, x \in G_{x}
$$

has a solution $\bar{z} \in G_{z}$ if and only if the inequalities

$$
W_{p, q}(\bar{z}, x) \leq W_{p, q}(\bar{z}, \bar{x}) \leq W_{p, q}(z, \bar{x})
$$

hold for all $z \in G_{z}, x \in G_{x}$, and $x \neq 0$.

Proof. Assume that the inequality $W_{p, q}(\bar{z}, x) \leq-\gamma\|x\|^{2 p}$ holds for a vector $\bar{z} \in G_{z}$. Then we have

$$
W_{p, q}(\bar{z}, x)<0=W_{p, q}(\bar{z}, \bar{x})=W_{p, q}(z, \bar{x})
$$

and the necessity of the assertion is proved.

Now we assume that (3.7) is true. Clearly,

$$
W_{p, q}(\bar{z}, x) \leq 0=W_{p, q}(\bar{z}, \bar{x})=W_{p, q}(z, \bar{x})
$$

It is matter of direct verification to prove that the set $F_{q}(x), x \in G_{x}$, is a bounded convex closed (a convex compact) subset of $\mathbb{R}^{n}$. Moreover, we deal with continuous functions. 
Hence,

$$
\begin{aligned}
W_{p, q}(\bar{z}, x) & \leq 2 p \max _{x \in G_{x}} \max _{y \in F_{q}(x)}\left\{\sum_{i=1}^{m}\left(l_{i}, x\right)^{2 p-1}\left(l_{i}, y\right)\right\} \\
& =\sum_{i=1}^{m}\left(l_{i}, \tilde{x}\right)^{2 p-1}\left(l_{i}, A \tilde{x}\right) \leq-\tilde{\gamma}\|\tilde{x}\|^{2}
\end{aligned}
$$

where $\tilde{\gamma}>0, \tilde{x} \in G_{x}$, and $A \in \mathscr{B}_{q}$. For $x \in G_{x}, x \neq 0$, we have

$$
-\tilde{\gamma}\|\tilde{x}\|^{2}=-\tilde{\gamma}\|x\|^{2} \frac{\|\tilde{x}\|^{2}}{\|x\|^{2}} \leq-\tilde{\gamma}\|x\|^{2} \min _{x \in G_{x}} \frac{\|\tilde{x}\|^{2}}{\|x\|^{2}} .
$$

Let $\gamma:=\tilde{\gamma} \min _{x \in G_{x}}\left(\|\tilde{x}\|^{2} /\|x\|^{2}\right)$. We obtain

$$
W_{p, q}(\bar{z}, x) \leq-\gamma\|x\|^{2}
$$

for all $x \in G_{x}, x \neq 0$. The proof is finished.

In other words, the pair $(\bar{z}, \bar{x}) \in G_{z} \times G_{x}$ is a saddle point of the function $W_{p, q}(\cdot, \cdot)$. After this investigation, we come back to the question leading to the construction of the Lyapunov function $V_{p}(\cdot, \cdot)$ for the given system (1.1) (or system (1.4)). This problem is now reduced to an auxiliary problem of mathematical programming, namely, to the saddle points problem described by inequalities (3.7). Note that in practice we define $G_{z}:=\left\{z \in \mathbb{R}^{N(p)} \mid\|z\| \leq \Gamma_{z}\right\}$. The constant $\Gamma_{z}$ can be determined, for instance, by a maximal machine number. We can also obtain the similar result for weakly asymptotic stable systems.

Theorem 3.2. Let $V_{p}(\cdot, \cdot)$ be the Lyapunov function from the class of homogeneous forms of order $2 p, p \in \mathbb{N}$, for system (1.1). The inequality

$$
W_{p, q}^{-}(z, x) \leq-\gamma\|x\|^{2 p}, \quad \gamma>0, x \in G_{x}
$$

has a solution $\bar{z} \in G_{z}$ if and only if the inequalities

$$
W_{p, q}^{-}(\bar{z}, x)<W_{p, q}^{-}(\bar{z}, \bar{x}) \leq W_{p, q}^{-}(z, \bar{x})
$$

hold for all $z \in G_{z}, x \in G_{x}$, and $x \neq 0$.

The proof of this theorem is analogous to the proof of Theorem 3.1.

\section{On the asymptotic stability of difference inclusions}

In this section, we study the initial-value problem for difference inclusions

$$
x(k+1) \in F_{q}(x(k)), \quad x(0)=0,
$$

where $k=0,1, \ldots$, and the multifunction $F_{q}(\cdot)$ is determined in Section 1 . The definition of asymptotic stability of the zero solution $x(\cdot) \equiv 0$ of $(4.1)$ is analogous to Definition 2.1. In particular, as in the case of the initial-valued problem (1.1), the asymptotic stability of 
the zero solution of $(4.1)$ can be established by means of the Lyapunov function $V_{m, p}(\cdot, \cdot)$ which in this case will satisfy the inequality (see, e.g., [27])

$$
\max _{y \in F_{q}(x)} V_{m, p}(l, y)-\alpha V_{m, p}(l, x) \leq 0,
$$

for some $0<\alpha<1$. For system (4.1), we introduce the Lyapunov function $V_{p}(\cdot, \cdot)$ (see Section 3 ) and consider the inequality

$$
W_{p, q}(z, x):=\max _{y \in F_{q}(x)} V_{p}(z, y)-\alpha V_{p}(z, x) \leq 0
$$

for $z \in G_{z}$ and $x \in G_{x}$. Evidently, relation (4.3) implies the inequality "along solutions" of the discrete system (4.1), namely, the inequality

$$
W_{p, q}(z, x(k))=\max _{y \in F_{q}(x(k))} V_{p}(z, y)-\alpha V_{p}(z, x(k)) \leq 0 .
$$

The difference inclusion in (4.1) can be considered as a discretization scheme for (1.1) (see [9]).

THeOREM 4.1. Let $V_{p}(\cdot, \cdot)$ be the Lyapunov function from the class of homogeneous forms of order $2 p, p \in \mathbb{N}$, for system (4.1). The inequality (4.3) has a solution $\bar{z} \in G_{z}$ if and only if the inequalities

$$
W_{p, q}(\bar{z}, x) \leq W_{p, q}(\bar{z}, \bar{x}) \leq W_{p, q}(z, \bar{x})
$$

are fulfilled for all $z \in G_{z}$ and $x \in G_{x}, x \neq 0$.

Proof. Let $\bar{z} \in G_{z}$ be a solution of (4.3). Since

$$
V_{p}(z, \bar{x})=0, \quad z \in G_{z}
$$

and $F_{q}(\bar{x})=\{0\}$, we have $W_{p, q}(\bar{z}, \bar{x})=W_{p, q}(z, \bar{x})=0$. This implies that the saddle points inequalities (4.5) are fulfilled.

Now we assume that $(\bar{z}, \bar{x}) \in G_{z} \times G_{x}$ is a solution of (4.5). Since

$$
W_{p, q}(\bar{z}, \bar{x})=0
$$

we obtain $W_{p, q}(\bar{z}, x) \leq 0$ for $x \in G_{x}, x \neq 0$.

As is evident from the foregoing, the problem of constructing the Lyapunov function for system (4.1) with difference inclusions is also reduced to an auxiliary saddle points problem.

\section{Numerical aspects}

This section is devoted to the numerical treatment of the auxiliary saddle points problems given above. We solve the saddle points problem by application of the gradient-type algorithm. Note that the gradient method is one of the most popular first-order methods for solving problems of mathematical programming (see, e.g., [11, 32]). Let us consider 
the convex Lyapunov function $V_{p}(\cdot, \cdot)$ and the function $W_{p, q}(\cdot, \cdot)$ from Theorem 3.1 or Theorem 4.1. Evidently, $W_{p, q}(\cdot, \cdot)$ is a differentiable function. The following initial-value problems present a variant of gradient method for saddle points problem (see $[2,19])$ :

$$
\begin{array}{ll}
\dot{\xi}=\frac{\partial W_{p, q}(\zeta, \xi)}{\partial \xi}, & \xi(0)=0, \\
\dot{\zeta}=-\frac{\partial W_{p, q}(\zeta, \xi)}{\partial \zeta}, & \zeta(0)=0 .
\end{array}
$$

By $\dot{\xi}(\cdot), \dot{\zeta}(\cdot)$ we denote here the derivatives of the smooth functions $\xi(\cdot), \zeta(\cdot)$ with respect to $t \in \mathbb{R}$. We investigate the limit points of the solutions of (5.1) for $t \rightarrow \infty$. It is necessary to stress that the conditions of convergence of the gradient-based procedure (5.1) conventionally contain the requirements of convexity of the function $W_{p, q}(\cdot, \cdot)$ in $z$ and its concavity in $x$. In this case, the considered method (5.1) converges to the solution of the equivalent minimax problem (see, e.g., [38]). Note that the function $W_{p, q}(z, \cdot)$ is (in general) not concave in variable $x \in G_{x}$ for any $z \in G_{z}$. Nevertheless, for system (5.1) with the function $W_{p, q}(\cdot, \cdot)$ it will be established that all its solutions have a limit at $t \rightarrow \infty$.

Let $C \subseteq G_{z}$ be the set of all solutions of the inequality

$$
W_{p, q}(z, x) \leq-\gamma\|x\|^{2}
$$

for system (1.1) or the set of all solutions of the inequality (4.3) for system (4.1). If (5.2) or (4.3) is solvable, that is, $C \neq \varnothing$, then the closure $\bar{C}$ of the set $C$ will be the totality of all solutions of the corresponding inequalities. We now formulate the next result.

Theorem 5.1. Let $V_{p}(\cdot, \cdot)$ be the Lyapunov function from the class of homogeneous forms of order $2 p, p \in \mathbb{N}$, for system (1.1) or (4.1). Assume that the set

$$
D:=\left\{(\bar{z}, \bar{x}) \in G_{z} \times G_{x}: \bar{z} \in \bar{C}, \bar{x}=0\right\}
$$

is the set of all saddle points of the corresponding function $W_{p, q}(\cdot, \cdot)$. Then any solution $(\xi(\cdot), \zeta(\cdot))$ of the initial-value problem (5.1) satisfies the following limit relations: $\lim _{t \rightarrow \infty}(\xi(t), \zeta(t))=(\bar{x}, \bar{z})$, where $\bar{z} \in \bar{C}$ and $\bar{x}=0$.

Proof. See [19, Theorem 3].

Theorem 5.1 makes it possible to use the computational scheme (5.1) for finding saddle points of $W_{p, q}(\cdot)$. We apply the gradient-type method (5.1) to a class of the initialvalued problems (1.1). Let us consider the following nonlinear system (see [4]):

$$
\begin{gathered}
\dot{x}=\mathscr{A} x+\sum_{s=1}^{S} b_{s} \phi_{s}\left(\left(c_{s}, x\right), t\right), \quad x(0)=0, \\
\phi_{s}: \mathbb{R} \times \mathbb{R}_{+} \longrightarrow \mathbb{R}, \quad \phi_{s}(0, t)=0, \quad s=1, \ldots, S,
\end{gathered}
$$

where $S \in \mathbb{N}$ and $b_{s} \in \mathbb{R}^{n}, c_{s} \in \mathbb{R}^{n}$ are constant vectors. Assume that the nonlinear function $\phi_{s}(\cdot, \cdot)$ satisfies the conditions

$$
0 \leq \phi_{s}(\sigma, t) \sigma \leq K_{s} \sigma^{2} \quad \forall(\sigma, t) \in \mathbb{R} \times \mathbb{R}_{+}, s=1, \ldots, S
$$


with $0<K_{s}<\infty$. Evidently, a function $u_{s}(x):=\left(c_{s}, x\right)$ in $(5.4)$ can be interpreted as a linear feedback control function for the control system

$$
\dot{x}=\mathscr{A} x+\sum_{s=1}^{S} b_{s} \phi_{s}(u, t), \quad x(0)=0 .
$$

In this case, the system (5.4) is a closed-loop control system. One can rewrite (5.4) in the following equivalent form [4]:

$$
\dot{x} \in F_{\phi}(x), \quad x(0)=0,
$$

where

$$
F_{\phi}(x)=\left\{y \in \mathbb{R}^{n}: y=\mathscr{A} x+\sum_{s=1}^{S} b_{s} \mu_{s}\left(c_{s}, x\right), 0 \leq \mu_{s} \leq K_{s}, s=1, \ldots, S\right\} .
$$

It can be shown (see [4]) that (5.7) is a special case of (1.1) if

$$
A_{\nu}=\mathscr{A}+\sum_{s=1}^{S} h_{\nu_{s}} b_{s} c_{s}^{T}, \quad q=2^{S}
$$

where $h_{v_{s}}=0$ or $h_{v_{s}}=K_{s}, v=1, \ldots, q$, and $s=1, \ldots, S$. We now give an example of system (5.4).

Example 5.2. Consider the initial-value problem

$$
\begin{gathered}
\dot{x}_{1}=x_{2}, \quad x_{1}(0)=0, \\
\dot{x}_{2}=-2 x_{1}-x_{2}-\phi\left(x_{1}, t\right), \quad x_{2}(0)=0 .
\end{gathered}
$$

In this example, we have $S=1, c=(0,1), b=(0,-1)$, and the matrix

$$
\mathscr{A}=\left(\begin{array}{cc}
0 & 1 \\
-2 & -1
\end{array}\right)
$$

is a Hurwitz-type matrix (see, e.g., [22]). Assume that the nonlinear function $\phi(\cdot, \cdot)$ satisfies the inequalities $0 \leq \phi(\sigma, t) \sigma \leq K \sigma^{2}$, where $(\sigma, t) \in \mathbb{R} \times \mathbb{R}_{+}$and $K$ is a positive constant. For system (5.10), we compute the Lyapunov function $V_{1}(\cdot, \cdot)$ from the class of quadratic forms such that

$$
\left(\nabla V(z, x),\left(A x+K\left(\begin{array}{c}
0 \\
-x_{2}
\end{array}\right)\right)\right)<-\gamma\|x\|^{2}, \quad \gamma>0
$$

for all solutions of (5.10). Clearly, $p=1, n=2$, and $N(p)=3$. Let $K=3.73$. Using the gradient-type method (5.1) and the limit procedure from Theorem 5.1, we obtain

$$
V_{1}(z, x)=x_{1}^{2}+1.1005 x_{1} x_{2}+1.6513 x_{2}^{2}
$$

Note that the computed Lyapunov function $V_{1}(z, \cdot)$ is convex. 
Example 5.3. We examine the same system (5.10) and construct the Lyapunov function $V_{2}(\cdot, \cdot)$ from the class of the fourth order forms. Note that $p=2$ and $N(p)=5$. For $K=6.40$ we have

$$
V_{2}(z, x)=x_{1}^{4}+0.301 x_{1}^{3} x_{2}+0.620 x_{1}^{2} x_{2}^{2}+0.211 x_{1} x_{2}^{3}+0.101 x_{2}^{4}
$$

The gradient-type method (5.1) and the limit procedure from Theorem 5.1 in Examples 5.2 and 5.3 are implemented in $C$. We used the "numerical recipes in $C$ " package [34] and some author programs for this purpose.

Finally, we note that a discrete variant of (5.4) can be written as follows:

$$
x(k+1)=\Delta t\left(x(k)+A x(k)+\sum_{s=1}^{S} b_{s} \phi_{s}\left(\left(c_{s}, x(k)\right), k \Delta t\right)\right), \quad x(0)=0,
$$

where $k=0,1, \ldots$ and $\Delta t$ is a step size for the variable $t$ in (5.4). Evidently, this discrete system is equivalent to the following system:

$$
x(k+1) \in F_{\phi}(x(k)), \quad x(0)=0, \quad k=0,1, \ldots
$$

with the above-presented multifunction $F_{\phi}$.

\section{Concluding remarks}

In this paper, we establish the important role of saddle points problems in computing polynomial Lyapunov functions for a class of initial-value problems for differential and difference inclusions. We investigate a new numerical schema derived from a variant of the gradient method. This schema can also be treated as a practicable computational approach to the related problem of robust stability. The presented algorithm has usual advantages and disadvantages of a first-order numerical method. Note that the main idea of the algorithm can be combined not only with the gradient-type method but also with some other techniques for saddle points problem. It is conceivable that one can use the proposed computational approach and the corresponding results in studies of the socalled receding horizon control (see, e.g., $[14,26]$ ). Moreover, this approach can also be applied to some other classes of continuous and discrete control systems governed by differential and difference inclusions.

\section{Acknowledgment}

The author is grateful to the anonymous referees for the valuable remarks, from which the final version of the paper greatly benefited.

\section{References}

[1] F. Amato, Robust Control of Linear Systems Subject to Time-Varying Parameters, Springer, Berlin, 2005.

[2] K. J. Arrov, L. Hurvitz, and H. Uzawa, Studies in Linear and Nonlinear Programming, Stanford University Press, Stanford, 1958. 
[3] J.-P. Aubin and A. Cellina, Differential Inclusions, Grundlehren der Mathematischen Wissenschaften, vol. 264, Springer, Berlin, 1984.

[4] V. Azhmyakov, A constructive method for solving stabilization problems, Discussiones Mathematicae. Differential Inclusions, Control and Optimization 20 (2000), no. 1, 51-62.

[5] B. R. Barmish, Necessary and sufficient conditions for quadratic stabilizability of an uncertain system, Journal of Optimization Theory and Applications 46 (1985), no. 4, 399-408.

[6] F. Bucci, Absolute stability of feedback systems in Hilbert spaces, Optimal Control (Gainesville, Fla, 1997) (W. H. Hager and P. M. Pardalos, eds.), Appl. Optim., vol. 15, Kluwer Academic, Dordrecht, 1998, pp. 24-39.

[7] K. Deimling, Multivalued Differential Equations, de Gruyter Series in Nonlinear Analysis and Applications, vol. 1, Walter de Gruyter, Berlin, 1992.

[8] A. L. Dontchev, Discrete approximations in optimal control, Nonsmooth Analysis and Geometric Methods in Deterministic Optimal Control (Minneapolis, Minn, 1993) (B. S. Mordukhovich and H. J. Sussmann, eds.), IMA Vol. Math. Appl., vol. 78, Springer, New York, 1996, pp. 59-80.

[9] A. L. Dontchev and F. Lempio, Difference methods for differential inclusions: a survey, SIAM Review 34 (1992), no. 2, 263-294.

[10] H. O. Fattorini, Infinite-Dimensional Optimization and Control Theory, Encyclopedia of Mathematics and Its Applications, vol. 62, Cambridge University Press, Cambridge, 1999.

[11] A. V. Fiacco and G. P. McCormick, Nonlinear Programming: Sequential Unconstrained Minimization Techniques, John Wiley \& Sons, New York, 1968.

[12] A. F. Filippov, Stability for differential equations with discontinuous and multivalued right-hand sides, Differentsial'nye Uravneniya 15 (1979), no. 6, 1018-1027, 1148-1149 (Russian).

[13] _ Differential Equations with Discontinuous Right-Hand Sides, Mathematics and Its Applications (Soviet Series), vol. 18, Kluwer Academic, Dordrecht, 1988.

[14] É. Gyurkovics, Receding horizon control for the stabilization of nonlinear uncertain systems described by differential inclusions, Journal of Mathematical Systems, Estimation, and Control 6 (1996), no. 3, 1-16.

[15] A. Halanay, Differential Equations: Stability, Oscillations, Time Lags, Academic Press, New York, 1966.

[16] D. Hinrichsen and A. J. Pritchard, Robustness measures for linear systems with application to stability radii of Hurwitz and Schur polynomials, International Journal of Control 55 (1992), no. 4, 809-844.

[17] M. Johansson, Piecewise Linear Control Systems, Lecture Notes in Control and Information Sciences, vol. 284, Springer, Berlin, 2003.

[18] R. E. Kalman, Lyapunov functions for the problem of Lur'e in automatic control, Proceedings of the National Academy of Sciences USA 49 (1963), 201-205.

[19] V. A. Kamenetskiy and Ye. S. Pyatnitskiy, An iterative method of Lyapunov function construction for differential inclusions, Systems \& Control Letters 8 (1987), no. 5, 445-451.

[20] V. L. Kharitonov, Asymptotic stability of an equilibrium position of a family of systems of linear differential equations, Differentsial'nye Uravneniya 14 (1979), 1483-1485 (Russian).

[21] M. Kisielewicz, Differential Inclusions and Optimal Control, Mathematics and Its Applications (East European Series), vol. 44, Kluwer Academic, Dordrecht; PWN—Polish Scientific Publishers, Warsaw, 1991.

[22] N. N. Krasovskiŭ, Stability of Motion. Applications of Lyapunov's Second Method to Differential Systems and Equations with Delay, Stanford University Press, California, 1963.

[23] A. I. Lourie and V. N. Postnikov, Concerning the theory of stability of regulating systems, Prikladnaya Matematika i Mekhanika 8 (1944), 246-248 (Russian).

[24] A. M. Lyapunov, The general problem of the stability of motion, International Journal of Control 55 (1992), no. 3, 521-790, translated into English by A. T. Fuller. 
[25] M. Mansour and B. D. O. Anderson, On the robust stability of time-varying linear systems, Stability Theory (Ascona, 1995) (R. Jeltsch and M. Mansour, eds.), Internat. Ser. Numer. Math., vol. 121, Birkhäuser, Basel, 1996, pp. 135-149.

[26] D. Q. Mayne and H. Michalska, Receding horizon control of nonlinear systems, IEEE Transactions on Automatic Control 35 (1990), no. 7, 814-824.

[27] A. P. Molchanov and Ye. S. Pyatnitskiy, Criteria of asymptotic stability of differential and difference inclusions encountered in control theory, Systems \& Control Letters 13 (1989), no. 1, 59-64.

[28] B. S. Mordukhovich, Discrete approximations and refined Euler-Lagrange conditions for nonconvex differential inclusions, SIAM Journal on Control and Optimization 33 (1995), no. 3, 882-915.

[29] Y. Orlov, Y. Lou, and P. D. Christofides, Robust stabilization of infinite-dimensional systems using sliding-mode output feedback control, International Journal of Control 77 (2004), no. 12, 11151136.

[30] P. C. Parks, A. M. Lyapunov's stability theory—100 years on, IMA Journal of Mathematical Control and Information 9 (1992), no. 4, 275-303.

[31] I. R. Petersen and C. V. Hollot, A Riccati equation approach to the stabilization of uncertain linear systems, Automatica. A Journal of IFAC 22 (1986), no. 4, 397-411.

[32] E. Polak, Optimization, Applied Mathematical Sciences, vol. 124, Springer, New York, 1997.

[33] V. M. Popov, Absolute stability of nonlinear systems of automatic control, Automatika i Telemechanika 22 (1961), 961-979 (Russian), translated in Automat Remote Control 22 (1962), 857-875.

[34] W. H. Press, S. A. Teukolsky, W. T. Vetterling, and B. P. Flannery, Numerical Recipes in C, Cambridge University Press, Cambridge, 1992.

[35] E. S. Pyatnitskii and L. B. Rapoport, The boundary of the domain of asymptotic stability of selectorlinear differential inclusions, and the existence of periodic solutions, Soviet Mathematics-Doklady 44 (1992), 785-790.

[36] B. Song and J. K. Hedrick, Simultaneous quadratic stabilization for a class of non-linear systems with input saturation using dynamic surface control, International Journal of Control 77 (2004), no. 1, 19-26.

[37] V. Veliov, Second-order discrete approximation to linear differential inclusions, SIAM Journal on Numerical Analysis 29 (1992), no. 2, 439-451.

[38] M. Walk, Theory of Duality in Mathematical Programming, Springer, Vienna; Akademie, Berlin, 1989.

[39] D. Wexler, On frequency domain stability for evolution equations in Hilbert spaces via the algebraic Riccati equation, SIAM Journal on Mathematical Analysis 11 (1980), no. 6, 969-983.

[40] V. A. Yakubovich, Solution of certain matrix inequalities occurring in the theory of automatic controls, Doklady Akademii Nauk. USSR 143 (1962), 1304-1307 (Russian), translated in Soviet Math. Dokl. 4 (1963), 620-623.

[41] V. I. Zubov, Mathematical Methods for the Study of Automatic Control Systems, Pergamon Press, New York; Jerusalem Academic Press, Jerusalem, 1962.

Vadim Azhmyakov: Institute for Automation Technology (IFAT) and Department of Electrical Engineering, Otto-von-Guericke University of Magdeburg, P.O. Box 4120, 39016 Magdeburg, Germany

E-mail address: vadim.azhmyakov@e-technik.uni-magdeburg.de 


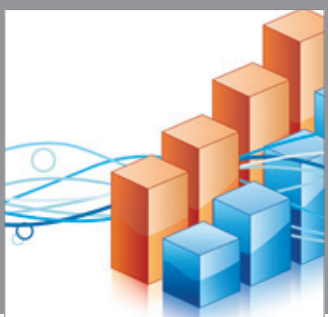

Advances in

Operations Research

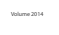

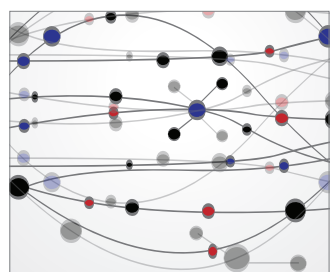

\section{The Scientific} World Journal
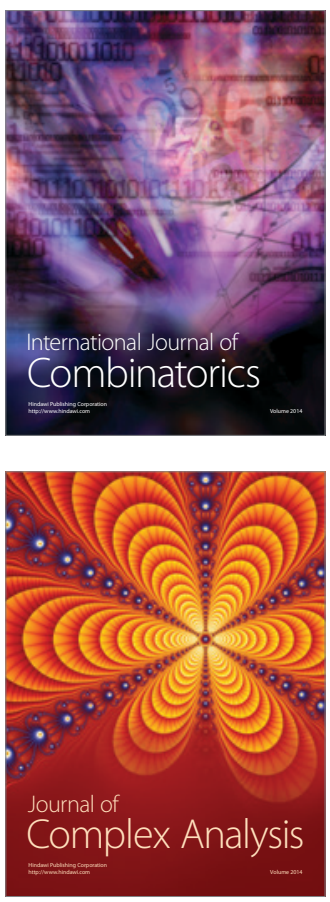

International Journal of

Mathematics and

Mathematical

Sciences
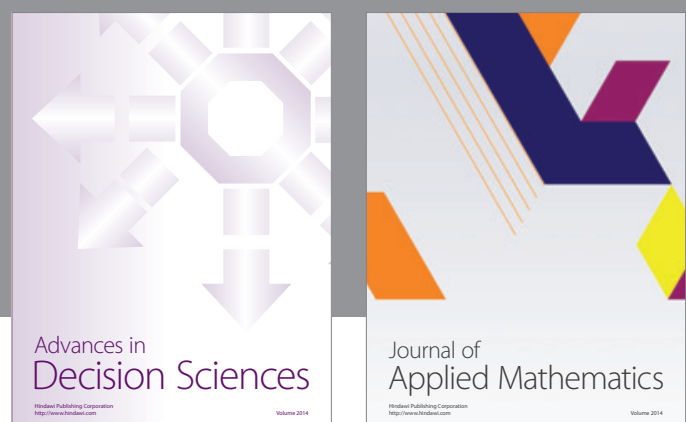

Journal of

Applied Mathematics
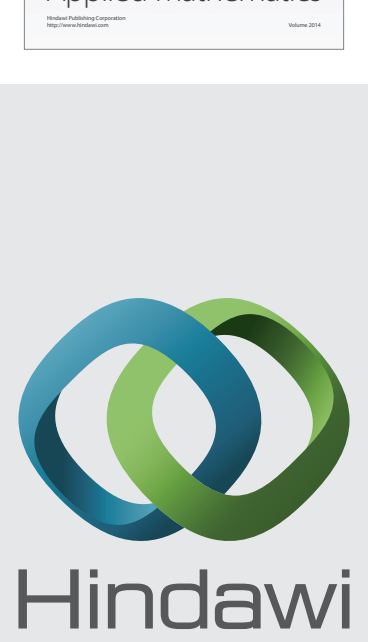

Submit your manuscripts at http://www.hindawi.com
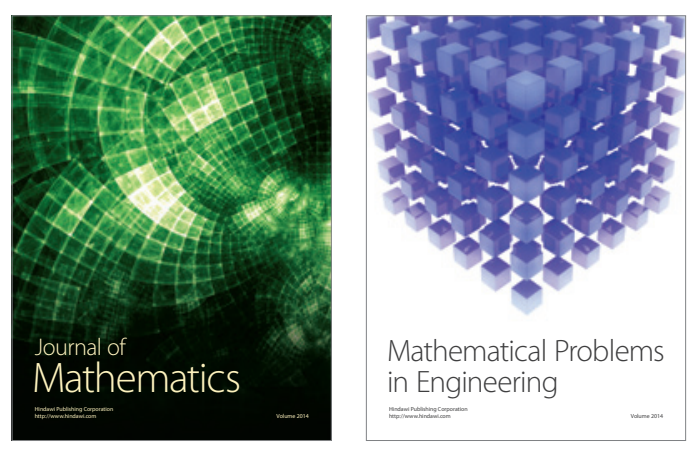

Mathematical Problems in Engineering
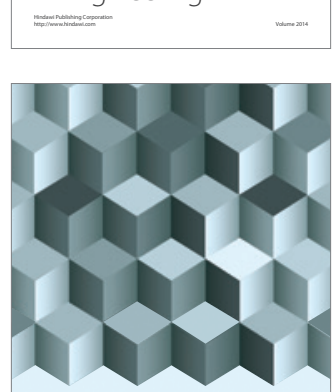

Journal of

Function Spaces
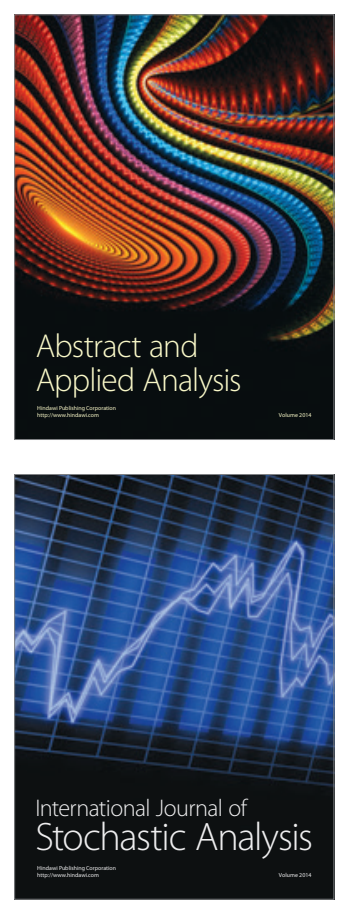

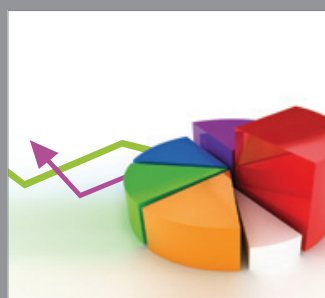

ournal of

Probability and Statistics

Promensencen
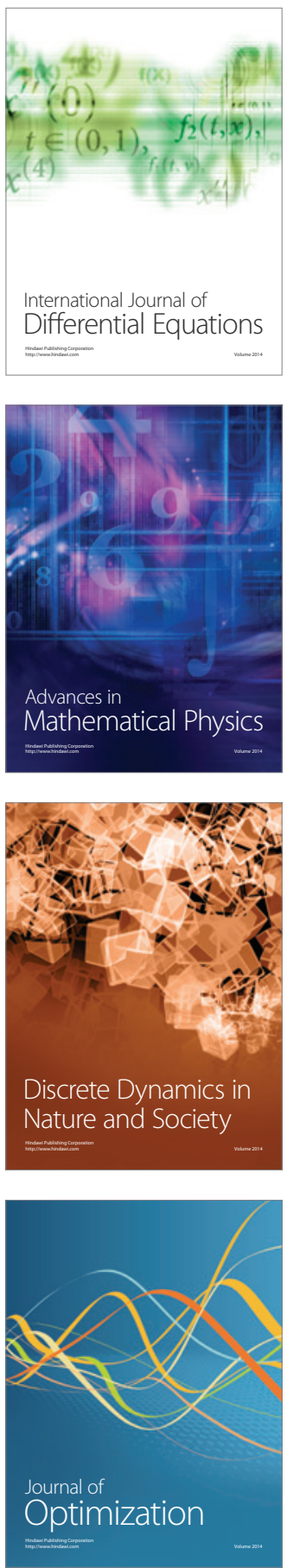